\title{
¿Empresarios innovadores? La eficacia interpelativa de los agronegocios en las identificaciones de los actores agropecuarios bonaerenses ${ }^{1}$
}

\author{
Dolores Liaudat ${ }^{2}$
}

\section{Resumen}

El objetivo de este artículo es examinar la influencia del discurso de los agronegocios, que es hegemónico en la esfera pública, en las identificaciones individuales económicolaborales de los actores del agro pampeano. Para ello tomamos como objeto de estudio a diferentes actores agropecuarios de la provincia de Buenos Aires. El corpus de análisis consiste en 42 entrevistas en profundidad desarrolladas en dos partidos bonaerenses (Ayacucho y Baradero) y complementariamente en 329 encuestas llevadas a cabo en 27 de las 30 zonas agroeconómicas homogéneas de dicha provincia. A partir de este estudio, damos cuenta que a pesar de que las transformaciones productivas que promueve el discurso de los agronegocios se han vuelto predominantes en el agro pampeano, en las identificaciones de los actores agropecuarios persisten posiciones subjetivas que adhieren a otras discursividades con presencia histórica en el sector, y que entran en tensión con el perfil identitario promovido por los agronegocios.

Palabras claves: agronegocios - identificaciones - hegemonía

\begin{abstract}
The aim of this article is to examine the influence of the agribusiness discourse, which is hegemonic in public sphere, in individual economic-labour identifications of the pampeanagricultural actors. We take as study object different agricultural actors of Buenos Aires province. The analysiscorpusconsists of 42 in-depth interviews conducted in two Buenos Aires counties (Ayacucho and Baradero) and 329 surveys carried out in 27 of the 30 homogeneous agro-economic zones of that province. From this study, we give an account that although the productive transformations promoted by the agribusinessdiscoursehave become predominant in the pampean agriculture, in the agricultural actors identifications persist subjective positions that adhere to other discursivities with historical presence in the sector, and that come into tension with the identity profile promoted by agribusiness.
\end{abstract}

Keywords: agribusiness - identifications hegemony

1 Trabajo recibido el 06/10/2018. Aceptado el 29/11/2018.

${ }^{2}$ Universidad Nacional de Quilmes. Universidad Nacional de La Plata. Consejo Nacional de Investigaciones Científicas y Técnicas. Contacto: doloresliaudat@yahoo.com.ar 


\section{Introducción}

A lo largo de la historia se han configurado en el agro argentino distintas discursividades que sostienen diferentes concepciones sobre el agro y su lugar en el desarrollo nacional. Específicamente en los discursos públicos sobre el agro pampeano conviven en relación de contradicción y/o complementación tres discursividades dominantes que se diputan la hegemonía: el discurso «liberal-conservador», centrado en defender la libertad de mercado y el derecho inalienable a la propiedad de la tierra; el «agrarista», enfocado en la denuncia de todas las formas de concentración y, por ende, de desigualdad social que se gestan en la estructura agraria, especialmente en torno al acceso a la tierra;y el «de los agronegocios», que pone énfasis en la innovación tecnológica y en el rol central del conocimiento para la generación de valor (Balsa, De Martinelli y Liaudat, 2017). Un importante número de estudios académicos han dado cuenta de cómo los agronegocios monopolizaron el discurso público sobre el campo en las últimas décadas, a través de su reiteración en los medios de comunicación, las políticas públicas o las entidades del sector (Balsa, 2008; Carniglia, 2011; Gras y Hernández, 2016; Liaudat, 2015).

Según este discurso, la figura tradicional del productor que integra todas las actividades «tranqueras adentro» (poseedor de tierras, de equipos y mano de obra propia) es una cuestión del pasado. La principal condición para ser parte de este sistema es abandonar estas pautas de acción consideradas tradicionales y asumir los rasgos del «empresario innovador» (Hernández, 2007 y 2009), cuyas características son las que se adjudican al espíritu capitalista en general: previsión, eficacia, ahorro, aprovechamiento del tiempo. Pero a ellas se agregan rasgos específicos de los discursos empresariales en esta nueva etapa del capital: innovación, flexibilidad, liderazgo, tercerización. Entre los soportes identitarios que son situados como incompatibles con este modelo y que son promovidos a abandonar se encuentra la relación simbólica con la tierra, la importancia de los saberes heredados y del trabajo directo. El discurso de los agronegocios construye esta identidad productiva en oposición a sujetos a los que considera retardatarios, como el chacarero o productor familiar (propias del discurso agrarista) y el estanciero terrateniente (figura clave en el discurso liberal-conservador), quienes históricamente han mantenido un vínculo simbólico y/o afectivo con la tierra (Hernández, 2007, p. 339). 
No es posible conocer la real capacidad hegemónica de este discurso sin ahondar en la eficacia interpelativa del mismo sobre sus principales destinatarios: los actoresagropecuarios.Eneste trabajo, desde una perspectiva teórica que articula los aportes de Antonio Gramsci, Louis Althusser y Stuart Hall, examinamos la influencia del discurso de los agronegocios -sin dejar de prestar atención a los otros discursos en disputa- en la construcción de las identificaciones económico-laborales de los actores agropecuarios de la provincia de Buenos Aires.

\section{Consideraciones teóricas: identificaciones, discurso e ideología}

En las últimas décadas, en el marco del denominado «giro lingüístico» y su atención sobre el lenguaje, el discurso y la narración (Arfuch, 2005), se ha cuestionado desde diferentes corrientes académicas la noción de una identidad originaria, unificada e integral. Pero este no ha sido un cuestionamiento solo teórico, sino que en este período se llevaron a cabo profundas transformaciones en las características de las identidades. En este trabajo adherimos a un número importante de autores de diferentes campos de estudio (Hall, 2003; Van Dijk, 1999; Laclau, 2009; Grimson, 2011) que han trabajado esta última dimensión a partir del concepto de «identificación». A través del mismo se pone énfasis en el carácter estratégico y posicional de las identidades, se las concibe como un proceso y no como una esencia. Este proceso inacabado, como toda práctica significativa, está sujeto al «juego de la diferencia».

A partir de esta perspectiva podemos pensar a las identidades construidas principalmente (aunque no solo) a través del discurso. En palabras de Hall (2003), es posible entender las identidades como puntos de sutura entre «por un lado, los discursos y prácticas que intentan interpelarnos', hablarnos o ponernos en nuestro lugar como sujetos sociales de discursos particulares y por otro, los procesos que producen subjetividades, que nos construyen como sujetos susceptibles de 'decirse'» (p. 20). La idea de «interpelación» proviene de Althusser (1970, p. 147), quien plantea que toda construcción ideológica interpela a los individuos como sujetos. En ese sentido es que el autor señala la inexistencia de sujetos pre-ideológicos; los individuos son interpelados como partícipes de diferentes papeles sociales y no son necesariamente coherentes en sus respuestas. 
Gramsci en los Cuadernos de la Cárcel abordó la disputa hegemónica en el plano de la constitución de subjetividades, señalando que la comprensión crítica de sí mismos se produce pues a través de la lucha de 'hegemonías' políticas, de direcciones contrastantes, primero en el campo de la ética, luego de la política, para llegar a una elaboración superior de la propia concepción de lo real. Es por esto que las construcciones identitarias no son solo individuales. Si bien los actores realizan reflexiones sobre sí mismos personales, éstas se producen a partir de discursos colectivos que los interpelan.

En ese artículo analizamos la eficacia interpelativa del discurso de los agronegocios sobre las identificaciones individuales de los actores agropecuarios. De las múltiples identificaciones que realizan los actores (sexual, cultural, étnica, etc.), en este caso nos centramos en el plano laboral, el cual consideramos tiene un lugar clave en la constitución del «sí mismo». Si bien la construcción identitaria no refiere solo al plano cognitivo (debido a que incluye determinadas prácticas, rutinas, objetos materiales), éste adquiere una gran centralidad a través del concepto de «representaciones sociales», puesto que los criterios de identificación no son tanto las acciones o los objetos en sí mismos, sino la construcción social en torno a los mismos. Es por eso que en este trabajo analizamos las identificaciones a través de las representaciones sociales de los actores agropecuarios.

\section{Estrategia metodológica}

La principal fuente de recolección y construcción de datos que sustentan las descripciones e hipótesis de este trabajo han sido entrevistas en profundidad desarrolladas con actores agropecuarios de dos localidades de la provincia de Buenos Aires: Ayacucho y Baradero. Éstas fueron realizadas entre fines del 2016 y comienzos del 2018. Complementariamente analizamos datos de la Encuesta sobre la Ideología de los Productores Rurales bonaerenses que hemos llevado a cabo en el 2013 con nuestro grupo de investigación.Elegimos como territorio de análisis a la provincia de Buenos Aires porque, siguiendo a Pucciarelli (1997), consideramos que su gran complejidad y extensión la hace altamente representativa de las diversas variantes productivas que se han desarrollado en la zona de producción agropecuaria más importante de Argentina: la región pampeana. 
Las entrevistas fueron realizadas con 42 actores de Ayacucho y Baradero. La opción por estos dos partidos corresponde, en primer lugar, a que poseen características productivas muy diferentes:son predominantemente ganadero y agrícola, respectivamente; lo que nos permite ganar en la representatividad del estudio. Al mismo tiempo, su elección corresponde a que son significativos como casos de estudio por las diversas características que en cada zona adquirió la estructura social agraria.Mientras en Ayacucho han predominado históricamente las explotaciones de gran extensión trabajadas principalmente en régimen de propiedad; en Baradero-un partido de fuerte tradición chacarera- han imperado las explotaciones de menor tamaño, teniendo mucho mayor peso el alquiler de tierras a terceros.

Las entrevistas asumieron una forma semi-estructurada. La muestra buscó incorporar diferentes tipos de actores agropecuarios que tengan presencia en los territorios locales, con el objetivo de realizar un análisis de carácter principalmente cualitativo que nos permita indagar en las subjetividades de los diversos actores que intervienen en el agro pampeano. Realizamos un muestreo por cuotas a partir de contactos personales e informantes clavesy se entrevistó a los siguientes tipos de actores en cada partido, distinguidos en función de su relación con los factores productivos (tierra, capital y trabajo): 1) rentistas, 2) empresarios medianos-grandes, 3) empresarios medianos, 4) empresarios pequeños $^{3}, 5$ ) empresarios contratistas, 6) contratistas familiares, 7) productores familiares, 8) productores unipersonales, 9) trabajadores de dirección, y 10) asesores profesionales.

La encuesta, por otra parte,contenía veintiocho preguntas abiertas y sesenta y seis preguntas cerradas y se planificó en base a una muestra de 396 casos, de los cuales finalmente se pudieron concretar 329 encuestas. La muestra no pretendía realizar aperturas por zonas al interior de la provincia; sin embargo, a fin de contar con una muestra representativa del conjunto y de las diversidades zonales se la organizó en base a las

\footnotetext{
${ }^{3}$ Realizamos la distinción entre empresarios medianos-grandes, medianos y pequeños a partir de dos criterios: las hectáreas que trabajan y el cálculo del ingreso neto de las explotaciones. Esta decisión se debe a que el valor de la tierra es muy diferente en ambos partidos y a que comparamos a empresarios de Ayacucho que poseen en propiedad la mayor parte de la tierra que trabajan con empresarios entrevistados de Baradero que casi no tienen tierra en propiedad. El ingreso neto de cada productor lo obtuvimos a partir de calcular el margen agrícola o ganadero de cada región menos los costos indirectos (alquileres de campo para los arrendatarios, y costos en impuestos y amortizaciones para los propietarios).
} 
30 zonas agroeconómicas homogéneas elaboradas por el INTA(hemos dejado de lado la zona del Delta y la del Gran Buenos Aires) (Álvarez et al, 2009; Mosciaro y Dimuro,2009). El número de casos es proporcional a la cantidad de explotaciones que cada zona tenía en el relevamiento censal del 2002; se presupuso que el proceso de concentración ha sido relativamente homogéneo en todas las zonas. Las zonas relevadas finalmente fueron 27 de las 30 zonas agroecológicas que incluimos en nuestra muestra debido a que hubo tres zonas en las que directamente no se logró realizar la encuesta (Zarate, Trenque Lauquen y Chillar). ${ }^{4}$

A falta de un listado muestral y ante las dificultades de implementación del trabajo de campo con los escasos recursos disponibles, no se pudo realizar un muestreo probabilístico y se implementó un muestreo por cuotas. El criterio general de selección de los casos era que deberían ser hombres o mujeres que estuvieran a cargo de una explotación agropecuaria, pero para garantizar la representatividad de los distintos tipos de productores se utilizaron cuotas sobre la base de cuatro variables estimadas a partir de la distribución presente en el último Censo Nacional Agropecuario fiable, realizado en el 2002: 1) por tamaño de las explotaciones, 2) por la tenencia del suelo, 3) según el uso del suelo y 4) una cuota de género.

En el siguiente mapa podemos observar el total de las zonas relevadas con ambos instrumentos metodológicos:

\footnotetext{
${ }^{4}$ Como hubo tres zonas en las que directamente no se logró realizar la encuesta, las ponderaciones no las realizamos sobre 396 casos, sino sobre 374 , que es el número total de casos, si restamos las encuestas que habíamos previsto realizar en estas tres zonas.
} 


\section{Mapa $\mathrm{N}^{\circ} 1$ : Zonas relevadas}

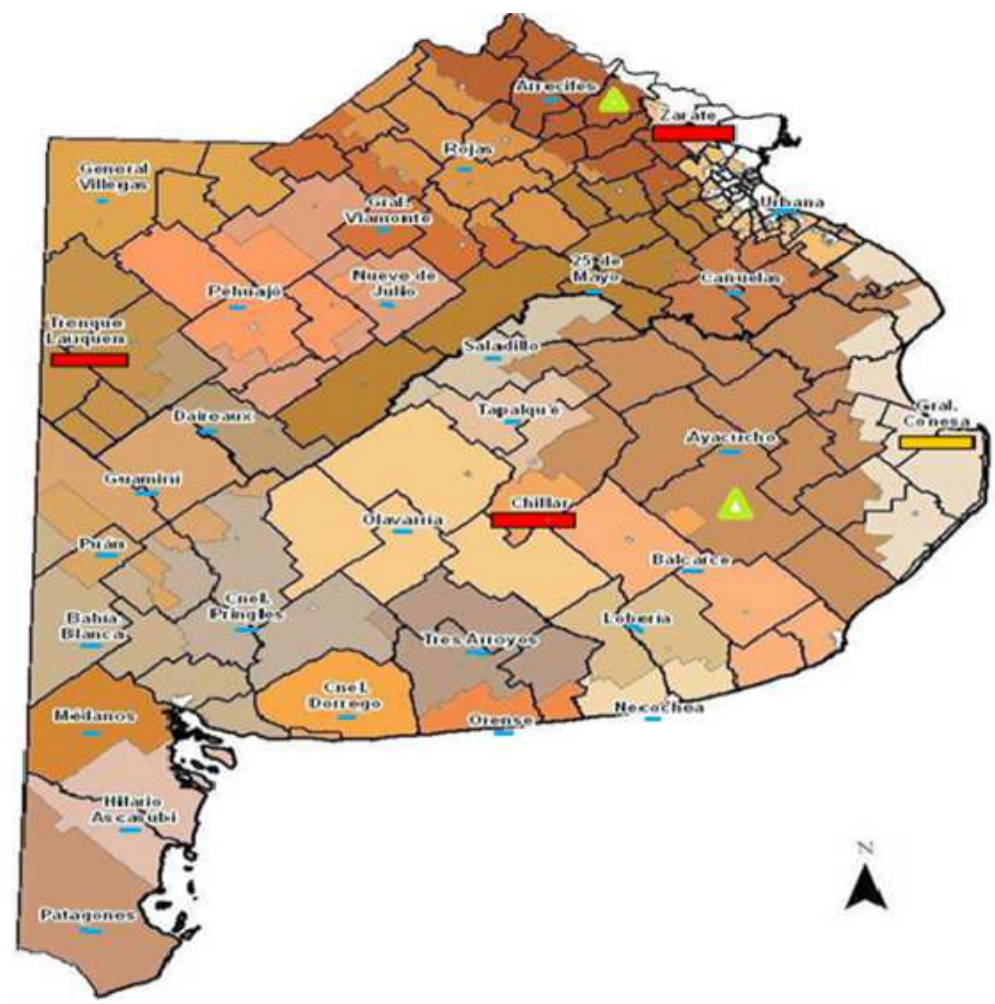

\section{Zonas encuestadas}

Zonas no encuestadas. En Zarate, Trenque Lauquen y Chillar por problemas logísticos no pudimos realizar el operativo.

Zona subencuestada. En General Conesa el relevamiento fue deficitario debido a que se pudo avanzar con la mitad de los casos previstos

Zonas entrevistadas

A partir de estos instrumentos se buscaron abordar dos grandes dimensiones de análisis. Mientras con las encuestas indagamos en el peso de las distintas discursividades de la esfera pública en las identificaciones de los actores agropecuarios bonaerenses, con las entrevistas analizamos la profundidad de la apropiación de dichas discursividades y la emergencia de discursos que se basan en la práctica de los actores. 


\section{¿Del productor chacarero y del estanciero al empresario innovador?}

El discurso de los agronegocios interpela a los actores agropecuarios como empresarios innovadores (Hernández, 2007; 2009). En esta figura se concentran las características del sujeto que protagonizaría este modelo. Uno de los rasgos centrales es el abandono de la referencia simbólica a la tierra, desarticulando la centralidad de esta última como sustrato identitario en su carácter de herencia (que condensa las trayectorias de los predecesores familiares), de saberes prácticos (el saber hacer aprendido en la experiencia y transmitido intergeneracionalmente) y/o de estatus simbólico que se afirman en un ser a través de la figura de «productor» (Manildo, 2009). La identificación como «productor» ha sido disputada por el discurso agrarista y el liberal-conservador a lo largo de la historia. Mientras el agrarismo ha buscado articular a dicho significante con un «saber hacer» específico y con una serie de delimitaciones en función del tamaño o la relación con la tierra («pequeños productores», «productores arrendatarios» $\mathrm{O}$ «productores chacareros»), el liberalismo-conservador se refiere a los «productores» a secas como un colectivo indiferenciado que puede hacer referencia, por ende, tanto a los que trabajan directamente la tierra como a los grandes estancieros (Balsa et al., 2017).

A partir de las entrevistas con los actores agropecuarios de Ayacucho y Baradero podemos observar que la construcción identitaria del discurso de los agronegocios tiene poca efectividad en los mismos. Teniendo en cuenta que en muchas ocasiones nuestros interlocutores se refieren a sí mismos de más de una manera, pudimos registrar a lo largo de las entrevistas ocho tipos de identificaciones individuales: 1) «soy del campo de toda la vida» (referencia a la biografía familiar), 2) «soy productor» (al interior de este grupo un porcentaje importante se definió como pequeño productor), 3) «soy chacarero», 4) «soy contratista», 5) «soy profesional» (referencia a los diferentes títulos universitarios que poseen los actores), 6) «soy asesor»; 7) «soy tradicional y moderno» (ubicamos en este grupo a quienes se refieren a sí mismos en la combinación de ambos rasgos), y 8) «soy empresario innovador». Mientras las dos primeras identificaciones son las que tuvieron mayor peso (más de la mitad de los entrevistados se presentó de ambas formas), la presentación del sí mismo como empresario innovador propia de la discursividad de los agronegocios fue sostenida por un solo entrevistado. 
Estas diversas modalidades de «narrativización del yo», como veremos a continuación, se insertan en otras discursividades con presencia histórica en el sector. A su vez, las identificaciones económico-laborales de los actores agropecuarios no se presentan en modo alguno como un sistema relacional coherente, sino que, por el contrario, las formas de referirse a sí mismos en relación con la actividad laboral en muchas ocasiones responden a discursividades diferentes. Como plantea Laplanche y Pontales (1985), «el ideal del yo está compuesto de identificaciones con ideales culturales que no son necesariamente armoniosos» (pp.208).

La identificación individual que tuvo mayor peso fue «soy del campo de toda la vida». Un grupo muy importante de entrevistados (26 de 42 en total) destacó como principal determinante de su identificación económico-laboral su biografía personal y familiar vinculada al campo desde su nacimiento. Entre quienes sostuvieron esta identificación, se destacan todos los rentistas y todos los empresarios medianos entrevistados y casi todos los contratistas familiares y empresariales (5 de los 6 entrevistados). Como plantea Hall (2003), «aunque parecen invocar un origen en un pasado histórico con el cual continúan en correspondencia, en realidad las identidades tienen que ver con las cuestiones referidas al uso de los recursos de la historia, la lengua y la cultura en el proceso de devenir y no de ser; no 'quiénes somos» o de 'dónde venimos', sino en que podríamos convertirnos, cómo nos han representado y cómo atañe ello al modo como podríamos representarnos» (pp. 17-18). En los siguientes ejemplos, podemos observar el uso del recurso de las historias personales en la construcción identitaria en frases como «soy nacido y criado en el campo» o «yo nací y mis padres nacieron en mi campo que todavía tenemos hoy»:

(...) yo nací y mis padres nacieron en mi campo, que todavía tenemos hoy. El campo debe andar arriba de los 100 años y mis hijos también nacieron ahí, hago agricultura y ganadería (Santiago, empresario mediano, Ayacucho).

(...) Soy nacido y criado en el campo, llevamos el campo adentro (Ariel, empresario mediano-grande, Baradero).

(...) soy hijo de productor agropecuario, viene de mi abuelo, bisabuelo, seguí en el rubro, hoy soy ingeniero agrónomo, soy de raíz agropecuaria más que nada contratista, hoy soy asesor en la cooperativa (Víctor, asesor, Baradero).

Mi viejo tuvo tambo toda la vida, me crie con la escuela del tambo, me fui a estudiar agronomía, arranqué hace diez años con equipos 
de pulverización y aparte hace un par de años arrancamos con la actividad productiva (Julián, empresario contratista, Ayacucho).

Como podemos ver en estos testimonios, los entrevistados comenzaron sus presentaciones autobiográficas en los relatos familiares y no en el plano profesional, como promueven los agronegocios. En «el campo» (en referencia a la explotación), se objetiva el esfuerzo de los antepasados («soy hijo de productor, viene de mi abuelo y mi bisabuelo») y se condensa el espacio de producción, reproducción y de adquisición de los principales saberes adquiridos («me crie en la escuela del tambo», «me crie en el campo»).

En estricta relación con su presentación ligada a la biografía familiar condensada en la explotación, gran parte de los entrevistados definieron su identificación económico-laboral como «productor» (25 de 42 casos). Es interesante destacar que no solo quienes son estrictamente productores (más allá de como organicen el trabajo) se refieren a sí mismos en primer lugar de esta manera, sino también contratistas, asesores y trabajadores de dirección, que tienen como actividad secundaria la producción propia o que directamente no la tienen. El imperativo implícito en esa inscripción inicial autobiográfica en la historia del campo familiar parecería ser un eco de un discurso pasado, basado en la apropiación del sujeto por la tierra que lo hereda y lo conmina a «convertirse en quien es» (Bourdieu, 1985, p.66). Este discurso en muchas ocasiones ya no tiene relación con la forma en que efectivamente organizan la producción o con la inserción laboral actual de los entrevistados, pero los sigue interpelando. Sin embargo, marca una tensión con el discurso de los agronegocios, ya que mientras este promueve la identificación de los actores agropecuarios a partir de la adquisición de determinados saberes profesionales, estos actores se afirman en la identidad de «productor» en relación a un vínculo especifico con la tierra (principalmente desde el saber hacer).

Entre los actores que se definieron como «productores», algunos se identificaron a sí mismos como «pequeño productor» ( 8 de los 25 que se definieron como productores). Entre ellos se encuentran casi todos los productores familiares, pequeños empresarios con historia familiar chacarera y algunos contratistas. Esta identificación denota el peso de la discursividadagrarista que señala la diferenciación de los productores por el tamaño de sus explotaciones. De la misma manera, encontramos la presencia de esta discursividad entre quienes se identificaron como «cha- 
careros». Esta identificación, de gran importancia política en la historia del agro pampeano, fue sostenida por un grupo no muy grande de entrevistados (6 en total, llamativamente sin asociación con un tipo de actor, cinco de ellos son de Baradero). En los siguientes relatos visualizamos, por un lado, la construcción de cadenas equivalenciales por medio de las cuales el significado del chacarero se articula a ser sembrador y/o arrendatario («como chacarero propio, como sembrador») y, por otro lado, la fragilidad para persistir de este sujeto en las condiciones actuales si no accede a las nuevas tecnologías ( «sin herbicida desaparezco, no soy chacarero») o si no alcanza la escala necesaria para sostenerse en la producción («un arrendatario como nosotros tiene que trabajar 400 o 500has, sino no te da»):

(...) como chacarero propio, como sembrador, soy muy chico. Aprendí a respetarla mucho a la chacra, mi economía no es fuerte y le tengo mucho respeto (...) sin herbicida desaparezco no soy chacarero. A mí me ponen como que enveneno a la gente entonces no soy chacarero si no enveneno a la gente, yo necesito rinde y para tener rinde necesito trabajar toda la tecnología de punta (Néstor, contratista familiar, Ayacucho).

Los campos son la mayoría alquilados, brindamos también servicios a terceros, pero poco, y tenemos muy poquito de ganadería. Tenemos muy poquito campo propio, mi abuelo también fue toda la vida chacarero (...) un arrendatario como nosotros tiene que trabajar 400 o 500 has sino no te da (Alberto, empresario mediano, Baradero)

Aunque pocos actores se definieron a sí mismos como chacareros, la mayoría de los que se presentaron a partir de su historia familiar ligada al campo y de los que lo hicieron como productores, construyeron sus identificaciones adscribiendo a una serie de rasgos que, según Muzlera (2009) corresponden a un habitus chacarero ${ }^{5}$ y que son propios del dis-

${ }^{5} \mathrm{El}$ autor, a partir del desarrollo de trabajo de campo en el sur de la provincia de Santa Fe, visibiliza claramente comomuchos actores agropecuarios experimentaron en las últimas décadas un conflicto interno entre los valores tradicionales chacareros y las características que deben ser adquiridas para subsistir en el actual modelo productivo. De esta manera lo sintetiza: «ser chacarero excede, por consiguiente a la mercancía producida y al proceso de producción; es un anclaje identitario, que está siendo transformado a fuerza de habitus desgarrados (Bourdieu, 1999). Muchas veces la permanencia depende del éxito que el heredero tenga en poder racionalizar las disposiciones heredadas, para adaptarse a las nuevas condiciones del capitalismo agrario» (Muzlera, 2009, p.152). 
curso agrarista. Estos refieren a un modo de organizar el trabajo y de vincularse con la tierra, un vínculo característico entre familia y explotación y a la recuperación del esfuerzo y del trabajo físico como medio de progreso.

Por otra parte, es importante destacar que entre quienes se definieron a sí mismos como contratistas (7 en total) se presentaron de esta forma luego de adjudicarse algunas de las otras identificaciones enunciadas. Por ejemplo: «soy del campo de toda la vida», «chacarero» y/o «productor» (aunque su principal fuente de ingresos sea la prestación de servicios). A su vez, la gran mayoría de los que tienen estudios superiores en temáticas referidas al agro se presentaron a sí mismos como ingenieros o técnicos agropecuarios ( 11 de los 15 que poseen estos estudios) y a su vez, seis entrevistados se autodefinieron como asesores profesionales (4 de los 8 que se desarrollan de esta manera como principal actividad laboral). Es interesante remarcar que principalmente los trabajadores de dirección destacaron en primer lugar su formación profesional como determinante en su identificación económico-laboral, mientras que los que son ingenieros y se desarrollan como primera actividad laboral como asesores, destacaron en primer orden su identificación como productores lo que denota la jerarquía que le dan a dicha identificación.

En los siguientes fragmentos visualizamos, por un lado, la identificación laboral de un trabajador de dirección centrada, en primer lugar, en los saberes profesionales («soy ingeniero en producción agropecuaria») y la presentación más adelante en su discurso de la actividad laboral que efectivamente realiza («me dedico a administrar empresas agropecuarias de terceros»); y, por otro lado, la primera identificación laboral de un asesor ligada al trabajo en la explotación familiar («soy productor agropecuario») y, en segundo orden, la identificación basada en un su saber profesional («pero también soy ingeniero»):

Tengo cincuenta y dos años, soy casado y tengo dos hijos, soy ingeniero en producción agropecuaria. Trabajo desde que tengo dieciocho años, estoy acá en Ayacucho desde el año '9l, hace ya veintiséis años. Me dedico a administrar empresas agropecuarias de terceros (Joaquín, trabajador de dirección, Ayacucho).

¿Cómo me defino? y...por ahí soy productor agropecuario, pero también soy ingeniero, o sea que por ahí al tener estudios universitarios me da capaz una visión más amplia, porque puedo ver otras cosas, puedo ver la actividad desde otra óptica porque también tengo mi profesión de agrónomo que es lo que, digamos, más me define. Pero 
por ahí vinculado al campo, estoy de chico. Porque soy hijo de productores agropecuarios de toda la vida, de cien años de familia y me críe en el campo, bah me críe en el pueblo, pero siempre fui a trabajar al campo con mi padre (Ezequiel, asesor, Ayacucho).

En este último relato advertimos una «dialéctica particular»(Gras y Hernández, 2009) entre, por un lado, la definición de su identidad por su pertenencia familiar y la relación con el campo como productor («soy hijo de productores agropecuarios de toda la vida, de cien años de familia y me crié en el campo»), y por otro lado, la voluntad de demarcarse de dicha identificación para forzar su propia identidad como portador de saberes profesionales que le otorgarían una visión más amplia («al tener estudios universitarios me da capaz que una visión más amplia, porque puedo ver otras cosas»). Esta dialéctica en la definición de la identidad que articula rasgos «tradicionales» (en relación al vínculo afectivo con la tierra y/o la explotación familiar) y «modernos» (la profesionalización) la encontramos solo en alrededor de un décimo de los entrevistados ( 5 de 42 en total) que se reconocieron a sí mismos como productores, pero asumieron también rasgos empresariales. Estos actores se definieron de esta manera argumentando el nivel de profesionalización con que llevan a cabo la actividad. En los siguientes relatos podemos observar algunos ejemplos:

Uno de los grandes cambios que ha ido habiendo es que se ha ido profesionalizando, salir de ser productor a ser empresario. La actividad de campo el que es productor se dedica a eso porque conoce, y después tenés el que es empresario y ve la actividad como un número y analiza más el número que el campo en sí. Hay un contenido muy emocional para poder diferenciar. Cuando uno no tiene lo sentimental piensa más en el negocio, la mayoría sigue con la parte vieja de lo que era el campo, dos marcos grandes. Yo me defino en un mix, yo me crie en el campo pero tuve también la otra parte de poder estudiar y pude ver que la parte administrativa, el número, es lo que mantiene activa las empresas, pero tengo la prueba piloto que es el campo de mi familia que me dio la capacitación que no me dio el estudio (Patricio, trabajador de dirección, Ayacucho).

(...) Estamos pasando de la agricultura familiar a la agricultura profesional, en el sentido de no haces lo que te gusta sino lo que tenés que hacer. Estamos peleando contra las grandes empresas que quieren entrar al sector en la parte que uno maneja (Walter, empresario contratista, Baradero). 
(...) pequeño pero empresario al fin, porque antes iba el chacarero vendía su producción y la cobraba. Hoy no, tiene que ser un empresario, tenés que tener un apoyo contable. Es una empresa por la cantidad de requisitos, la cantidad de impuestos hace que vos tenés que ser un empresario, te hace que estés pensando como evadir impuestos más que producir y eso te lleva a ser un empresario, por el volumen, que es muy grande (Alfredo, empresario pequeño, Baradero)

La noción de profesionalización refiere al proceso por medio del cual el cálculo y el conocimiento (en palabras de los entrevistados, ver en el campo «el negocio», «el número», «lo contable», el «riesgo») van reemplazando a un tipo de racionalidad donde aún pesaban elementos afectivos y valorativos (en palabras de los entrevistados «lo sentimental», «lo emocional», «lo que te gusta»). Es decir, hay un desplazamiento en términos de Weber (1996) de la racionalidad sustantiva por la racionalidad formal. Sin embargo, más allá de que estos actores resalten la adquisición de rasgos empresariales, es importante destacar que todos señalaron cierta tensión con esta transición identitaria. Esta se expresa en frases como «no haces lo que te gusta sino lo que tenés que hacer» o «la cantidad de requisitos, la cantidad de impuestos hace que vos tenés que ser un empresario». Estas palabras dan cuenta de una necesidad (del deber) de adaptarse a una nueva racionalidad para sobrevivir y no de la adopción activa de dichos rasgos en función de la ampliación de oportunidades como pregona el discurso de los agronegocios.

Este planteo típico de los agronegocios lo encontramos únicamente en el testimonio de un empresario mediano-grande de Baradero. De todos los entrevistados, este relato es el único que condensa varios de los rasgos identitarios del perfil empresarial que propaga el discurso de los agronegocios: flexibilidad, conocimiento, información y mirada global. La adopción de esta identificación empresarial se expresa en las palabras del entrevistado como una superación de la identificación como productor («no estar encasillado como productor») y la descripción de la actividad agropecuaria como un negocio donde los actores compiten con diferentes capitales («esto es un negocio que vos tenés ventajas o desventajas»):

(...) el hecho asociarme, ser flexible, abierto, por ahí no estar encasillado como productor. Hay productores menos flexibles, con su tierra, sus cosas, muchos se abrieron. Los tipos que crecieron son 
los que se abrieron, los que van adelantando, les van ganando al resto. Esto es un negocio, que vos tenés ventajas o desventajas con respecto a uno $(. .$.$) cada uno tienen una ventaja, uno siembra mejor$ porque tiene mejor tierra, y la ventaja comparativa mía es el conocimiento, el vender bien, es el estar informado, no hay mucha gente que opere en Chicago... y bueno yo lo hago todo el tiempo y eso te permite una diferencia (Agustín, empresario mediano-grande, Baradero).

Llamativamente la identidad netamente empresarial que expresa este relato no la encontramos en ningún otro de nuestros interlocutores. En las encuestas realizadas en el año 2013 en la provincia de Buenos Aires hallamos resultados similares a los de las entrevistas. Al comienzo de la misma les preguntamos «En relación con su ocupación laboral, ¿usted diría que es un...?», la respuesta era abierta y la hemos codificado en el análisis. Como veremos en la siguiente tabla, la representación de sí mismos como empresarios casi no tuvo presencia entre los entrevistados.

\section{Tabla $\mathrm{N}^{\circ} 1$. Formas de identificación individual de los productores agropecuarios}

\begin{tabular}{l|l|l}
\hline Identificación individual & Frecuencia & Porcentaje \\
Productor & 142 & $37,9 \%$ \\
Productor con identificación por actividad & 123 & $32,8 \%$ \\
Productor y otra identificación profesional & 35 & $9,3 \%$ \\
Productor por tamaño & 13 & $3,4 \%$ \\
Chacarero/arrendatario & 7 & $1,8 \%$ \\
Ingeniero & 11 & $2,9 \%$ \\
Trabajador & 11 & $2,9 \%$ \\
Asesor & 9 & $2,4 \%$ \\
Empresario & 6 & $1,6 \%$ \\
Encargado de producción & 4 & $1,0 \%$ \\
Emprendedor & 3 & $0,8 \%$ \\
Esclavo/explotado & 3 & $0,8 \%$ \\
Productor familiar & 1 & $0,2 \%$
\end{tabular}




\begin{tabular}{l|l|l}
\hline Identificación individual & Frecuencia & Porcentaje \\
No productor & 1 & $0,2 \%$ \\
Jubilado & 1 & $0,2 \%$ \\
Total & 370 & $98,9 \%$ \\
Perdidos & 4 & $1,1 \%$ \\
Total & 374 & $100 \%$ \\
\hline
\end{tabular}

Fuente:elaboración propia en base a la Encuesta de la ideología de los Productores Rurales bonaerenses (IESAC, 2013)

La gran mayoría de los encuestados (alrededor de un $85 \%$ ) se identificó individualmente como «productor». Entre ellos, más del 38\% se definió meramente como productor agropecuario, el 33,2\% como productor con diferenciación por la actividad que realizan (productor ganadero/agrícola/tambero), un 9,4\% se definió como productor y una segunda identificación entre las que se encuentran ingeniero, administrador, consignatario de hacienda y contratista; y el 3,5\% se definió como productor con diferenciación por tamaño (identificándose principalmente como pequeños productores). A su vez, podemos observar que muy pocos se reconocieron a sí mismos en función de su relación con la tierra como chacareros y/o arrendatarios $(1,8 \%)$ y en función del lugar del trabajo directo propio y/o familiar tras la categoría de productor o agricultor familiar $(0,3 \%$ se reconocieron en esta figura). Esto da cuenta de la pérdida de relevancia de las identificaciones «chacareros» o «estancieros» de enorme centralidad en las discursividades que disputaron históricamente en el agro pampeano, como de la poca eficacia de la variante actual del discurso agrarista en torno a la categoría de «agricultor familiar» en los actores del agro bonaerense. Por último, un número muy pequeño se identificó con la interpelación de los agronegocios como empresarios (solo el 1,6\% de los entrevistados) o como emprendedores $(0,8 \%)$.

Si analizamos la relación entre las identificaciones individuales y el tamaño de la explotación del actor entrevistado, no encontramos vínculos claros. Es posible señalar únicamente algunas asociaciones leves. Por un lado, el lugar de los empresarios y productores medianos (que trabajan entre 500 y 1000has) entre quienes se definen como productores en función de la actividad que realizan (casi el $44 \%$ de los entrevistados de 
dicha escala de producción respondieron de esta forma). Por otro lado, el mayor porcentaje de quienes se definieron como «pequeños productores» o «chacareros» lo encontramos entre los productores de menor escala (que trabajan 50 o menos has). A pesar de que en esta categoría social la mayor fuerza la tiene la definición como productores a secas o como productores en función de la actividad, alrededor de un $18 \%$ de estos actores se refirió con estas identificaciones propias del discurso agrarista. Los escasos entrevistados que se definieron con la identidad empresarial que promueven los agronegocios se repartieron entre las diferentes escalas de tamaño.

Por otra parte, en la encuesta se les pidió a los entrevistados que dijeran cuán de acuerdo estaban con una serie de frases típicas de las tres principales discursividades sobre cuestiones agrarias que predominan en el debate público. En líneas generales, cada frase buscaba tener un efecto interpelativo «seductor»; es por ello que estaban formuladas de manera tal de concitar la adhesión a las mismas con términos que contuviesen motivos argumentales que favorecieran ese acuerdo. De las frases que eran propias de la discursividad de los agronegocios las que mayor peso tuvieron son las que referían al cambio tecnológico. En contraste, aquella frase que refería a la identidad empresarial de los actores que protagonizan la producción agropecuaria obtuvo mucha menos adhesión, como podemos ver en la siguiente tabla:

Tabla No2. Nivel de acuerdo con la frase: Los productores agropecuarios ya no son chacareros sino que son empresarios

\begin{tabular}{llll}
\hline Nivel de acuerdo & Frecuencia & Porcentaje \\
\hline \multirow{4}{*}{ Válidos } & Totalmente & 103 & $27,4 \%$ \\
& Medianamente & 103 & $27,6 \%$ \\
& Solo un poco & 76 & $20,3 \%$ \\
& Nada & 91 & $24,4 \%$ \\
Perdidos & Notal & 373 & $99,7 \%$ \\
Total & 1 & $0,3 \%$ \\
\hline
\end{tabular}

Fuente:elaboración propia en base a la Encuesta de la ideología de los Productores Rurales bonaerenses (IESAC, 2013) 
Solo un $27,5 \%$ de los encuestados estuvo totalmente de acuerdo con esta frase, mientras que alrededor de un $28 \%$ estuvo medianamente de acuerdo, un 20,3\% solo un poco de acuerdo y el $24,5 \%$ estuvieron nada de acuerdo. Teniendo en cuenta el tipo de ejercicio, que en términos generales tendía a producir adhesión a las frases por el tipo de formulación, el hecho de que casi un cuarto de los entrevistados responda no estar nada de acuerdo da cuenta de un posicionamiento consciente en contra de esta idea que intentan instalar con fuerza los agronegocios.

\section{Reflexiones finales}

El discurso de los agronegocios construye todo un bagaje conceptual que interpela a los actores agropecuarios a redefinir su identidad en pos de generar consenso sobre esta nueva etapa de avance del capital sobre el agro pampeano. En este trabajo pudimos visibilizar la poca eficacia de esta propuesta ideológica, centrada en lo empresarial y en la superación de los «límites» de lo rural, en las identificaciones de los actores agropecuarios.

En sus identificaciones individuales, la mayoría de los actores adhirió a las posiciones subjetivas que construyen otras discursividades. La mayor parte se refirió a sí mismo en relación con su historia familiar vinculada a la actividad agropecuaria y al «campo» en general (con identificaciones como «soy del campo de toda la vida», «nacido y criado en el campo») y como productor agropecuario. A su vez, un porcentaje importante dentro de estos últimos se definiócomo «pequeño productor», una forma de identificarse influenciada por el discurso agrarista. Sin embargo, a pesar de la adscripción entre los actores entrevistados a una serie de rasgos característicos del «habitus chacarero» y del discurso agrarista (el vínculo entre familia y explotación, relación extra-mercantil con la tierra y valoración del esfuerzo), tuvieron escasa presencia las identificaciones personales como «chacarero» y/o «productor familiar», dos construcciones identitarias de clase que han tenido históricamente una gran importancia política en el agro pampeano.

Más allá del escaso peso de estas identificaciones, hemos observado que los actores agropecuarios aún se sienten interpelados por una discursividad que refiere a una forma de organizar la producción que implicaba un «modo de vida» que paradójicamente ya muchos de ellos no sostienen. Algunos de nuestros interlocutores hace tiempo que 
abandonaron la vida en el campo y el trabajo directo en la explotación (delegando gran parte de las tareas), pero se siguen reconociendo en la identificación como «productor» ligada al trabajo, el sacrificio y la relación directa con la naturaleza. Otros actores nunca llegaron a desempeñarse laboralmente de esa forma en el campo y tampoco a vivir allí, pero también se sienten interpelados por un discurso sobre la identidad del «hombre de campo» atravesado por estos tópicos. Este último es el caso, por ejemplo, de las entrevistados más jóvenes, hijos de varias generaciones de productores (quienes se desempeñan como trabajadores de dirección, asesores e incluso pequeños empresarios agropecuarios y/o contratistas) que, aunque nunca vivieron en el campo y/o el trabajo directo fue muy reducido (ayudan al padre los fines de semana o en las vacaciones), mantienen una discursividad donde el vínculo con la tierra los define.

No obstante, es necesario destacar que se evidencia en estas nuevas generaciones la convivencia de este discurso con tópicos propios de los agronegocios, en una transición atravesada por tensiones que se expresan en la presencia de rasgos «tradicionales» $\mathrm{y}$ «modernos» en sus identificaciones laborales y en la enunciación de la «necesidad» de adaptarse a un nueva racionalidad para sobrevivir en esta etapa del capitalismo agropecuario pampeano.

\section{Referencias bibliográficas}

Althusser, L. (1970). Ideología y aparatos ideológicos del Estado. Buenos Aires, Argentina: Nueva Visión.

Álvarez, R., Leavy, S. y Marino, M. (2009). Zonas Agroeconómicas Homogéneas Buenos Aires Norte. Buenos Aires, Argentina: INTA.

Balsa,J. (2008). La ideología de los productores rurales pampeanos. En: Realidad Económica, 237 (10), 55-79.

Balsa, J., De Martinelli, G y Liaudat, D. (2017). La ideología de los productores rurales bonaerenses en la actualidad. EnG. De Martinelli y M. Moreno (Comps.).Cuestión agraria y agronegocios en la región pampeana. Tensiones en torno a la imposición de un modelo concentrador. Bernal, Argentina: Universidad Nacional de Quilmes.

Bourdieu, P. (1985). ¿Qué significa hablar? Economía de los intercambios Linguiisticos. Madrid, España: Akal.

Carniglia, E. (2011). Las ruralidades de la prensa. Agronegocio, tecnología 
y agrarismo. Río Cuarto, Argentina: Universidad Nacional de Río Cuarto.

Gramsci, A. (1981-1999). Cuadernos de la Cárcel. México, México: Era.

Gras, C. (2009). El nuevo empresariado agrario: sobre la construcción y los dilemas de sus organizaciones. EnC. Gras y V. Hernández (Coords.).La Argentina rural De la Agricultura familiar a los agronegocios, pp. 215-236. Buenos Aires, Argentina: Biblos.

Gras, C. y Hernández, V. (2016). Radiografía del nuevo campo argentino. Del terrateniente al empresario transnacional. Buenos Aires, Argentina: Siglo XXI.

Grimson, A. (2011). Los limites de la cultura. Critica de las teorias de la identidad. Buenos Aires, Argentina: Siglo XXI.

Hall, S. (2003). Introducción: ¿Quién necesita identidad? En S. Hall y P. Du Gay (Comps).Cuestiones de identidad cultural, pp. 13-39. Buenos Aires-Madrid, Argentina-España: Amorrortu.

Hernández, V. (2007). El fenómeno económico y cultural del boom de la soja y el empresariado innovador. Desarrollo Económico, 47 (187), 331-365.

Hernández, V. (2009). La ruralidad globalizada y el paradigma de los agronegocios en las pampas gringas. En C. Gras y V. Hernández (Coords.).La Argentina rural De la Agricultura familiar a los agronegocios, pp. 39-64. Buenos Aires, Argentina: Biblos.

Laclau, E. (2009). La razón populista. Buenos Aires, Argentina: Fondo de Cultura Económica.

Laplanche, J. y Pontalis, J.B. (1985) «Post-scriptum», in Fantasmeoriginaire, fantasmes des origines, origines du fantasme. Paris, Francia: Hachette, textes du XX Siècle.

Liaudat, D. (2015). La construcción hegemónica de las entidades técnicas en el agro argentino: análisis de los discursos de AAPRESID y AACREA en la última década. Mundo Agrario, 16 (32). Recuperado de http://www.mundoagrario.unlp.edu.ar/article/view/ MAvl6n32a04

Manildo, L. (2009). Las fronteras sociales de la identidad. El impacto de las transformaciones recientes en el agro pampeano sobre la construcción del «nosotros» social. En XXVII Congreso de la Asociación Latinoamericana de Sociología. VIII Jornadas de Sociología de la Universidad de Buenos Aires. Asociación Latinoamericana de So- 
ciología, Buenos Aires. Recuperado dehttp://cdsa.aacademica.org/ 000-062/457.pdf

Mosciaro, M. y Dimuro, V. (2009). Zonas Agroeconómicas Homogéneas Buenos Aires Sur. Buenos Aires, Argentina: INTA.

Muzlera, J. (2009). Transformaciones, continuidades y tensiones en el mundo chacarero. La herencia en la pampa gringa. En C.Gras y V. Hernández (Coords.).La Argentina rural De la Agricultura familiar a los agronegocios, pp. 135-152. Buenos Aires, Argentina: Biblos.

Pucciarelli, A. (1997). Estructura agraria de la pampa bonaerense. Los tipos de explotaciones predominantes en la provincia de Buenos Aires. En O. Barsky y A. Pucciarelli.El agro pampeano. El fin de un periodo(pp. 206-287). Buenos Aires, Argentina: Facultad Latinoamericana de Ciencias Sociales-Universidad de Buenos Aires.

Van Dijk, T. A (1999). Ideología. Una aproximación multidisciplinaria. Barcelona, España: Gedisa.

Weber, M. (1996). Economía y Sociedad. México, México: Fondo de Cultura Económica. 